\section{Cobertura do exame citopatológico do colo do útero na cidade de São Leopoldo, Rio Grande do Sul, Brasil}

\author{
Coverage of Pap smear tests in the city of São \\ Leopoldo, Rio Grande do Sul State, Brazil
}

\begin{abstract}
This cross-sectional study aimed to verify the coverage of Pap smear tests and associated factors in a cluster sample of women 20 to 60 years of age in the city of São Leopoldo, Rio Grande Sul State, Brazil, in 2003. Two outcomes were analyzed: Pap smear in the previous three years and Pap smear never performed. Among 867 women, 741 (85.5\%; 95\%CI: 83.1-87.8) had a Pap smear in the previous three years, 60 (6.9\%; 95\% CI: 5.28.6) were late with the test, and 66 (7.6\%; 95\%CI: 5.8-9.4) had never done a Pap smear. Poisson regression showed significant association with: income, age, skin color, and marital status. Although coverage was high, risk factors for uterine cervical cancer were not associated with performing the test.
\end{abstract}

Vaginal Smears; Uterine Cervical Neoplasms; Health Services Coverage
Deise Karine Muller 1

Juvenal Soares Dias-da-Costa 1,2

Anna Maria Hecker Luz $^{3}$

Maria Teresa Anselmo Olinto 1

\section{Introdução}

No Brasil, as taxas de mortalidade por câncer do colo uterino têm se mostrado moderadamente altas, mas estáveis, a taxa em 1979 foi 4,97/100 mil 1 e em 2005: 4,81/100 mil (Sistema de Informações sobre Mortalidade; http://www.datasus. gov.br, acessado em 13/Nov/2007). Em 2005, na população feminina, a mortalidade proporcional do câncer de colo uterino foi de $6,6 \%$, sendo que em 2005 causou a morte de 4.506 mulheres. O câncer do colo do útero é a quarta causa de morte entre os óbitos por neoplasia na população feminina no Rio Grande do Sul 2.

O câncer do colo do útero tem sido descrito como uma afecção iniciada com transformações intra-epiteliais progressivas que pode evoluir para uma lesão cancerosa invasora, em 10 a 20 anos 3 . Assim, pode ser considerado uma neoplasia evitável devido à longa fase pré-invasiva, quando suas lesões precursoras podem ser detectadas, pela disponibilidade de triagem através do exame citopatológico de Papanicolaou e pela possibilidade de tratamento eficaz das lesões 4.

Em 1998, o Ministério da Saúde lançou o Programa Nacional de Controle do Câncer do Colo do Útero e de Mama, visando a mobilização e busca ativa das mulheres alvo, coleta do exame citopatológico do colo uterino e tratamento dos casos positivos de forma eqüitativa 5 . O programa ressaltou a realização do exame citopatológico em todas as mulheres com vida sexual ativa de tal 
forma que após dois exames anuais consecutivos negativos, a cada três anos um novo exame fosse feito. Salientou também que a prevenção do câncer em estágio inicial fosse maior em mulheres com idade entre 35 e 49 anos, especialmente entre aquelas que nunca realizaram o exame citopatológico 6 . Alguns estudos foram realizados no Brasil mostrando a cobertura do exame. Os resultados referentes ao Brasil da Pesquisa Mundial de Saúde, realizada em 2003, mostraram que $65 \%$ das mulheres de 18 e 69 anos tinham realizado o exame de Papanicolauou nos três anos anteriores 7. A Pesquisa Nacional por Amostra de Domicílios de 2003 (PNAD 2003) 8 apontou que a cobertura do exame foi de $68,7 \%$ entre as mulheres com mais de 24 anos.

Contudo, em estudos epidemiológicos têm se adotado como ponto de corte para realização do exame de Papanicolaou os últimos três anos, partindo-se do pressuposto estabelecido por Day 9 , o qual demonstrou que o risco cumulativo para desenvolver câncer cérvico-uterino invasivo nas idades entre 20 e 64 anos em países ocidentais foi reduzido em $84 \%$ para mulheres rastreadas a cada cinco anos e em $91 \%$ para aquelas examinadas a cada três anos. Sendo que as mulheres submetidas anualmente ao procedimento teriam uma proteção adicional de $2 \% 9$.

Portanto, este estudo teve por objetivos verificar a cobertura e fatores associados à realização do exame preventivo de câncer do colo do útero nos últimos três anos ou alguma vez na vida na população de mulheres com vida sexual ativa, de 20 a 60 anos residentes na zona urbana de São Leopoldo, Rio Grande do Sul, Brasil, durante o ano de 2003.

\section{Material e métodos}

O presente estudo fez parte do projeto de pesquisa Condições de Saúde das Mulheres: Estudo de Base Populacional na Região do Vale do Rio dos Sinos, estudo transversal com múltiplos desfechos realizado entre março e novembro de 2003.

O estudo foi realizado em São Leopoldo, município situado na região do Vale do Rio dos Sinos, localizado a aproximadamente $30 \mathrm{~km}$ da capital, Porto Alegre. No último censo demográfico do Instituto Brasileiro de Geografia e Estatística (IBGE), a população era de 193.547 habitantes, sendo que deste total, 98.781 (51,1\%) eram mulheres 10 .

O tamanho da amostra foi calculado levandose em consideração vários desfechos previstos no projeto de base populacional. Optou-se pelo maior tamanho da amostra. Foram levados em consideração alguns parâmetros para o cálculo, nível de confiança de $95 \%$, poder de $80 \%$, razão de não expostos: expostos, estabelecida em 1:3. Assim foi realizada uma amostra por conglomerados, sendo sorteados quarenta setores censitários entre os 270 existentes na zona urbana da cidade de São Leopoldo. Cada um desses setores teve o primeiro quarteirão sorteado juntamente com o ponto de partida. Desta forma, para completar a população necessária foram visitados 36 domicílios em cada setor. Após a finalização da coleta dos dados, foram encontradas $1.084 \mathrm{mu}$ lheres de 20 a 60 anos, destas 58 (5,3\%) foram classificadas como perdas e recusas, sendo incluídas no estudo 1.026 pessoas entrevistadas. Foram incluídas na análise 867 mulheres que referiram ter vida sexual ativa.

Foram aplicados questionários padronizados, pré-codificados e pré-testados. Os entrevistadores receberam treinamento para a coleta dos dados. Em 10\% da amostra foi realizado o controle de qualidade da coleta de dados através de questionário simplificado.

Foram analisados dois desfechos. Inicialmente, tentou-se verificar associações entre as mulheres com o exame atrasado, ou seja, que não tinham realizado o citopatológico de câncer do colo do útero nos últimos três anos. O segundo desfecho procurou caracterizar as mulheres que nunca haviam realizado o exame de Papanicolaou. Foram exploradas associações entre os desfechos e algumas variáveis sócio-econômicas, demográficas, obstétricas e ginecológicas e de utilização de serviços de saúde.

As variáveis sócio-econômicas incluídas no estudo foram: classe econômica, escolaridade em anos de estudo e renda familiar per capita. As classes econômicas foram definidas com base na tabela de classificação da Associação Nacional de Empresas de Pesquisa (ANEP) 11, a partir da posse de bens materiais, da presença de empregados domésticos nas residências e escolaridade do chefe da família.

As variáveis demográficas incluídas na análise foram: idades agrupadas, cor da pele observada pelo entrevistador e estado civil. Entre as variáveis obstétricas e ginecológicas foram incluídas: número de filhos, menarca, uso de preservativos nas relações sexuais e história familiar de câncer de colo uterino. As variáveis de utilização dos serviços de saúde foram: número de consultas médicas realizadas no último ano e tipo de serviço utilizado (sistema público ou seguros e sistema privado).

Os dados foram digitados duas vezes através do programa Epi Info (Centers for Disease Control and Prevention, Atlanta, Estados Unidos). A análise univariada foi realizada utilizando o programa SPSS versão 11.0 (SPPS Inc., Chicago, Es- 
tados Unidos). A amostragem em múltiplos estágios exigiu o cálculo do efeito de delineamento com as respectivas correções das medidas de efeito, intervalos de 95\% de confiança (IC95\%) e testes do qui-quadrado calculados através do programa Stata versão 7.0 (Stata Corp., College Station, Estados Unidos). Uma vez que os desfechos tiveram prevalências elevadas optou-se pela análise ajustada através da regressão de Poisson. Na regressão de Poisson a medida de efeito é a razão de prevalência evitando-se resultados superestimados decorrentes das medidas de odds 12 . A análise multivariada foi realizada segundo modelo hierarquizado 13 , conforme a Figura 1. As variáveis que ingressaram no modelo tiveram valor de $\mathrm{p}<20 \%$ na análise bruta.

\section{Resultados}

Entre as 867 mulheres incluídas no estudo, 741 (85,5\%; IC95\%: 83,1-87,8) tinham realizado o exame citopatológico do colo uterino nos últimos três anos, 60 (6,9\%; IC95\%: 5,2-8,6) estavam com o procedimento atrasado e 66 (7,6\%; IC95\%: 5,8$9,4)$ nunca o haviam realizado.

Quanto às principais características da amostra, $40,3 \%$ das mulheres se encontravam na classe econômica C, 43,5\% tinham menos de sete anos de estudo e $77,4 \%$ apresentavam renda igual ou inferior a três salários mínimos. Em relação às variáveis demográficas, $29,1 \%$ das mulheres estavam na faixa etária dos 40 aos 49 anos, 84,7\% foram classificadas como brancas e 73,6\% estavam casadas ou em união. As variáveis ginecológicas e obstétricas mostraram que $67,9 \%$ das mulheres tinham de 1 a 3 filhos, 45,2\% manifestaram a ocorrência de menarca entre 12 e 13 anos, 70,9\% não usavam preservativos nas relações sexuais e $90 \%$ não apresentavam história familiar de câncer de colo de útero. Quanto à utilização dos serviços de saúde, $37,1 \%$ das mulheres haviam realizado cinco ou mais consultas médicas no último ano e $32,2 \%$ referiram usar o sistema público (Tabela 1).

A amostragem foi por conglomerados exigindo o cálculo do efeito de delineamento. Para a primeira variável analisada como desfecho (exame citopatológico de colo uterino não realizado nos últimos três anos), o efeito do delineamento foi de 1,11 , sendo considerado para a análise bruta e ajustada.

A análise bruta mostrou associação estatística entre exame atrasado com classe econômica, escolaridade e renda. As mulheres inseridas nas classes C, D e E apresentaram maior percentual de exame não realizado nos últimos três anos do que aquelas das classes A e B. A associação reve-
Figura 1

Modelo hierarquizado para regressão de Poisson.

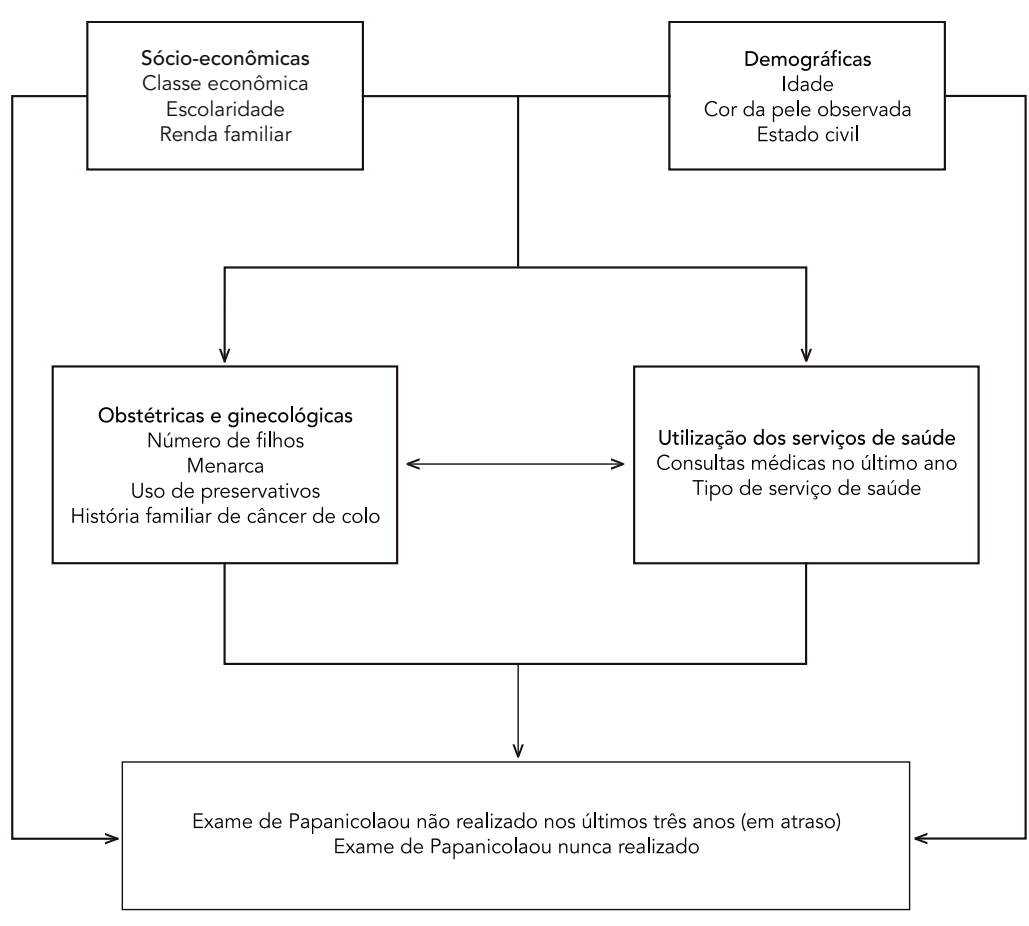

lou tendência linear, a cada nível de mudança de classe econômica aumentava a prevalência de mulheres com exame atrasado. As mulheres com até 11 anos de escolaridade tinham uma probabilidade maior do que três vezes de realizarem menos o exame do que as inseridas na categoria de referência. Nas mulheres com renda mais baixa também foram constatadas maiores prevalências de exames atrasados. As mulheres entre 40 e 60 anos, classificadas como brancas e com um a três filhos foram mais examinadas. Enquanto aquelas que não se consultaram com médico no ano anterior à pesquisa e que utilizaram o serviço público de saúde apresentaram maior prevalência de exame atrasado. As mulheres viúvas, separadas ou solteiras, aquelas com menarca a partir dos 12 anos de idade apresentaram maiores prevalências de exames atrasados. Por outro lado, as mulheres que não usavam preservativos nas relações sexuais e com história familiar realizaram mais o exame. Contudo, estado civil, menarca, uso ou não de preservativos nas relações sexuais e história familiar de câncer do colo do útero não apresentaram significância estatística para não realização do exame (Tabela 1). 
Exame de Papanicolaou não realizado nos últimos três anos (exame atrasado) de acordo com algumas variáveis sócio-econômicas, demográficas, ginecológicas e de utilização de serviços de saúde. São Leopoldo, Rio Grande do Sul, Brasil, 2003.

\begin{tabular}{|c|c|c|c|c|c|c|c|}
\hline \multirow[t]{2}{*}{ Variável } & \multirow[t]{2}{*}{$\mathrm{n}$} & \multirow[t]{2}{*}{$\%$} & \multicolumn{2}{|c|}{ Exame atrasado } & \multirow{2}{*}{$\begin{array}{l}\text { Razão de } \\
\text { prevalência }\end{array}$} & \multirow[t]{2}{*}{ IC95\% } & \multirow{2}{*}{$\begin{array}{l}\text { Valor } \\
\text { de } p\end{array}$} \\
\hline & & & $\mathrm{n}$ & $\%$ & & & \\
\hline Classe econômica * & & & & & & & $<0,001$ \\
\hline$A$ e $B$ & 298 & 34,5 & 17 & 5,7 & 1,00 & & \\
\hline C & 349 & 40,3 & 53 & 15,2 & 2,66 & $1,58-4,50$ & \\
\hline$D$ e $E$ & 218 & 25,2 & 56 & 25,7 & 4,50 & $2,69-7,53$ & \\
\hline Escolaridade (anos) & & & & & & & 0,007 \\
\hline 15 ou mais & 103 & 11,9 & 5 & 4,9 & 1,00 & & \\
\hline $12-14$ & 244 & 28,1 & 32 & 13,1 & 2,70 & $1,08-6,74$ & \\
\hline $8-11$ & 143 & 16,5 & 26 & 18,2 & 3,75 & $1,49-9,43$ & \\
\hline $5-7$ & 208 & 24,0 & 35 & 16,8 & 3,47 & $1,40-8,58$ & \\
\hline $0-4$ & 169 & 19,5 & 28 & 16,6 & 3,41 & $1,36-8,56$ & \\
\hline Renda (salários mínimos) * & & & & & & & $<0,001$ \\
\hline$>6,01$ & 74 & 8,5 & 7 & 9,5 & 1,00 & & \\
\hline $3,01-6,00$ & 122 & 14,1 & 5 & 4,1 & 0,43 & $0,14-1,32$ & \\
\hline $1,01-3,00$ & 351 & 40,5 & 46 & 13,1 & 1,39 & $0,65-2,95$ & \\
\hline$<1,01$ & 320 & 36,9 & 68 & 21,3 & 2,25 & $1,08-4,69$ & \\
\hline Idade (anos) * & & & & & & & 0,001 \\
\hline $20-29$ & 249 & 28,7 & 50 & 20,1 & 1,00 & & \\
\hline $30-39$ & 232 & 26,8 & 31 & 13,4 & 0,67 & $0,44-1,00$ & \\
\hline $40-49$ & 252 & 29,1 & 32 & 12,7 & 0,63 & $0,42-0,95$ & \\
\hline $50-60$ & 134 & 15,5 & 13 & 9,7 & 0,48 & $0,27-0,86$ & \\
\hline Cor da pele & & & & & & & 0,003 \\
\hline Branca & 734 & 84,7 & 99 & 13,5 & 1,00 & & \\
\hline Não branca & 133 & 15,3 & 27 & 20,3 & 1,51 & $1,30-2,21$ & \\
\hline Estado civil & & & & & & & 0,44 \\
\hline Casadas ou em união & 638 & 73,6 & 89 & 13,9 & 1,00 & & \\
\hline Viúvas, separadas ou solteiras & 229 & 26,4 & 37 & 16,2 & 1,16 & $0,81-1,65$ & \\
\hline Número de filhos & & & & & & & $<0,001$ \\
\hline Nenhum & 165 & 19,0 & 31 & 18,8 & 1,00 & & - \\
\hline $1-3$ & 589 & 67,9 & 70 & 11,9 & 0,63 & $0,43-0,93$ & \\
\hline 4 ou mais & 113 & 13,0 & 25 & 22,1 & 1,18 & $0,74-1,88$ & \\
\hline Idade da menarca (anos) & & & & & & & 0,62 \\
\hline $8-11$ & 167 & 19,3 & 19 & 11,4 & 1,00 & & \\
\hline $12-13$ & 392 & 45,2 & 60 & 15,3 & 1,35 & $0,83-2,18$ & \\
\hline 14 ou mais & 308 & 35,5 & 47 & 15,3 & 1,34 & $0,81-2,21$ & \\
\hline Uso do preservativo & & & & & & & 0,47 \\
\hline Sim & 252 & 29,1 & 40 & 15,9 & 1,00 & & \\
\hline Não & 615 & 70,9 & 86 & 14,0 & 0,88 & $0,62-1,24$ & \\
\hline História familiar de câncer de colo do útero & & & & & & & 0,09 \\
\hline Não & 780 & 90,0 & 118 & 15,1 & 1,00 & & \\
\hline Sim & 87 & 10,0 & 8 & 9,2 & 0,61 & $0,31-1,20$ & \\
\hline Consultas médicas no último ano & & & & & & & $<0,001$ \\
\hline 5 ou mais & 322 & 37,1 & 39 & 12,1 & 1,00 & & \\
\hline $3-4$ & 187 & 21,6 & 18 & 9,6 & 0,79 & $0,47-1,35$ & \\
\hline $1-2$ & 249 & 28,7 & 27 & 10,8 & 0,90 & $0,56-1,42$ & \\
\hline Nenhuma & 109 & 12,6 & 42 & 38,5 & 3,18 & $2,18-4,64$ & \\
\hline Tipo de serviço de saúde & & & & & & & $<0,001$ \\
\hline Sistema público & 97 & 32,2 & 18 & 18,6 & 1,00 & & \\
\hline Seguros e sistema privado & 204 & 67,8 & 14 & 6,9 & 0,37 & $0,19-0,71$ & \\
\hline
\end{tabular}

* Teste de tendência linear $<0,001$. 
Na análise multivariada através da regressão de Poisson seguindo o modelo hierárquico para o exame preventivo do câncer de colo uterino não realizado nos últimos três anos, as mulheres pertencentes às classes sociais C, D e E e aquelas que não se consultaram com médico no último ano continuaram apresentando maior prevalência de exame atrasado. Observou-se que as mulheres a partir dos trinta anos de idade e que procuravam os serviços privados foram mais submetidas ao exame. As mulheres classificadas como cor da pele não branca e com quatro ou mais filhos apresentaram maiores prevalências de exames atrasados. Entretanto, as variáveis: idade, cor da pele, número de filhos e tipo de serviço de saúde não apresentaram significância estatística (Tabela 2).

Na análise bruta do outro desfecho, "mulheres que nunca realizaram o exame preventivo de Papanicolaou", também se levou em conta o efei- to de delineamento para apresentação das medidas de efeito e respectivos intervalos de confiança (efeito = 1,02).

As mulheres nas classes econômicas C, D e E apresentaram maior prevalência de exame nunca realizado. Foi observado teste de tendência linear significativo em relação à renda familiar. As mulheres nas duas menores categorias de menor renda também tinham maiores prevalências de nunca realizar o exame, apesar de os intervalos de confiança não evidenciarem diferenças nas estimativas.

Quanto à distribuição por idade, verificouse que a partir dos cinqüenta anos a prevalência de exame nunca realizado foi menor do que a encontrada na categoria de base (20 a 29 anos). Foi constatado teste de tendência linear significativo, mostrando que à medida que aumentava a idade, diminuía o percentual de mulheres com o exame nunca realizado.

Tabela 2

Análise multivariada para exame de Papanicolaou não realizado nos últimos três anos de acordo com algumas variáveis sócio-econômicas, demográficas, ginecológicas e de utilização de serviços de saúde. São Leopoldo, Rio Grande do Sul, Brasil, 2003.

\begin{tabular}{|c|c|c|c|}
\hline Variável & Razão de prevalência & IC95\% & Valor de $p$ \\
\hline Classe econômica * & & & $<0,001$ \\
\hline$A$ e $B$ & 1,00 & & \\
\hline C & 2,52 & $1,49-4,26$ & \\
\hline $\mathrm{D}$ e $\mathrm{E}$ & 4,07 & $2,40-6,90$ & \\
\hline Idade (anos) * & & & 0,24 \\
\hline $20-29$ & 1,00 & & \\
\hline $30-39$ & 0,76 & $0,51-1,14$ & \\
\hline $40-49$ & 0,76 & $0,51-1,14$ & \\
\hline $50-60$ & 0,60 & $0,34-1,07$ & \\
\hline Cor da pele * & & & 0,32 \\
\hline Branca & 1,00 & & \\
\hline Não branca & 1,22 & $0,83-1,80$ & \\
\hline Número de filhos ** & & & 0,16 \\
\hline Nenhum & 1,00 & & \\
\hline $1-3$ & 0,60 & $0,27-1,34$ & \\
\hline 4 ou mais & 1,15 & $0,46-2,90$ & \\
\hline Consultas médicas no último ano ** & & & $<0,001$ \\
\hline 5 ou mais & 1,00 & & \\
\hline $3-4$ & 0,76 & $0,45-1,28$ & \\
\hline $1-2$ & 0,89 & $0,56-1,40$ & \\
\hline Nenhuma & 2,71 & $1,85-3,96$ & \\
\hline Tipo de serviço de saúde ** & & & 0,91 \\
\hline Sistema público & 1,00 & & \\
\hline Seguros e sistema privado & 0,96 & $0,47-1,96$ & \\
\hline
\end{tabular}

* Variáveis que ingressaram no primeiro nível e que foram ajustadas entre si;

** Ajustadas para a variável classe econômica. 
As mulheres classificadas como as viúvas, separadas ou solteiras, que não tiveram filhos, que não consultaram no último ano e que procuraram preferencialmente o sistema público de saúde apresentaram maiores prevalências de exame preventivo do câncer do colo do útero nunca realizado. As mulheres com cor da pele classificada como não branca e com menarca depois dos 12 anos de idade apresentaram maiores prevalências de exames atrasados. Já as mulheres com história familiar de câncer do colo uterino e que não usavam preservativos nas relações sexuais foram mais submetidas ao exame. Todavia, as variáveis: cor da pele, menarca, uso de preservativo nas relações sexuais e história familiar de câncer do colo do útero não apresentaram significância estatística em relação ao exame nunca realizado (Tabela 3).

$\mathrm{Na}$ análise multivariada, o modelo de regressão de Poisson para exame nunca realizado apontou que as mulheres das classes sociais C, $\mathrm{D}$ e E apresentaram maiores prevalências do que as das classes A e B. As mulheres acima de trinta anos apresentaram menor prevalência de exame nunca realizado, mas aquelas que não se consultaram no ano anterior ao da pesquisa apresentavam maior probabilidade de não realizar o Papanicolaou (Tabela 4).

As variáveis: cor da pele observada, estado civil, número de filhos, história familiar, e tipo de serviço de saúde utilizado não mostraram associação com o desfecho (Tabela 4).

\section{Discussão}

Os estudos transversais podem fornecer valiosas informações para a organização e gestão de sistemas de saúde, desde que representem a população de referência. O presente estudo incluindo amostra da população feminina de São Leopoldo apresentou em cada faixa etária a mesma distribuição proporcional verificada no censo de 2000 (IBGE. Censo Demográfico de 2000. http:/ / ww.ibge. gov.br), caracterizando sua representatividade.

A cobertura do exame citopatológico do colo uterino nos últimos três anos entre as mulheres incluídas no estudo foi de 85,5\% (IC95\%: 83,1$87,8)$. Em Pelotas, no Rio Grande do Sul, estudos transversais de base populacional têm descrito as prevalências de cobertura de realização deste exame. Em 1992, encontraram-se 65\% (IC95\%: 61,9-68,0) de cobertura para o exame 14; em 2000 a cobertura foi 72\% (IC95\%: 69,6-74,8) 15; em 2001 verificaram-se 68,8\% (IC95\%: 66,2 a 71,4) 16; e em 2003 encontraram-se 81\% (IC95\%: 79,0-83,0) 17. Na cidade de São Paulo, um estudo realizado em 2000 mostrou que 77,3\% (IC95\%: 74,1-80,5) das
1.050 mulheres entrevistadas tinham feito o exame nos últimos três anos 18 . Foi realizada uma revisão sistemática da literatura sobre cobertura do exame de Papanicolaou no Brasil no período entre 1966 e 2004 19, nenhum estudo incluído atingiu cobertura superior à verificada em São Leopoldo. Além disso, parece ter alcançado o nível de cobertura preconizado pelo Ministério da Saúde, pelo Instituto Nacional de Câncer (INCA) e pela Organização Mundial da Saúde (OMS) ao redor de $80 \% 6,20$.

No presente estudo verificou-se que $66 \mathrm{mu}$ lheres $(7,6 \%$; IC95\%: 5,8-9,4) nunca haviam realizado o exame de Papanicolaou. Outro estudo transversal de base populacional realizado na cidade de Rio Grande, Rio Grande do Sul, constatou que entre 1.302 mulheres, 57\% (IC95\%: 54,3$59,7)$ nunca haviam realizado o exame ${ }^{21}$. Já em Pelotas, Quadros et al. 16 verificaram que 21,3\% das mulheres nunca haviam realizado o exame. Esse resultado merece considerações, pois em virtude da longa história natural do câncer de colo de útero e da proteção que o rastreamento pode proporcionar, a OMS preconiza que o exame citopatológico seja prioritariamente realizado ao menos uma vez, nas mulheres entre 35 e 40 anos de idade em países que precisam implantar o controle em curto prazo 6 .

Assim, as coberturas encontradas em São Leopoldo foram elevadas, embora não se tenha testado a validade das informações coletadas. Discute-se se as mulheres não se sentem pressionadas a responderem positivamente a respeito de procedimentos preventivos, superestimando as reais coberturas de procedimentos 22 .

A faixa etária de maior risco para mortalidade por câncer do colo do útero é dos 40 aos 60 anos 3 . A análise do presente estudo mostrou que as mulheres de 40 a 49 anos foram as mais examinadas, entretanto na faixa etária de 50 a 60 anos, as prevalências de exame atrasado ou nunca realizado não foram estatisticamente diferentes do que as apresentadas pelo grupo mais jovem.

Apesar das coberturas elevadas, a análise das características associadas à não realização do procedimento mostrou que a presença de fatores reconhecidamente de risco para surgimento do câncer de colo uterino não aumentou a cobertura do exame de Papanicolaou. Seguindo esse critério, fatores de risco para o desenvolvimento da doença como história familiar de câncer de colo uterino ou não utilização de preservativos nas relações sexuais não apresentaram significância estatística. Outros estudos também mostraram que as políticas de planejamento e captação das mulheres a serem rastreadas pelo exame não estão levando em consideração reconhecidos fatores de risco para a doença 15,17 . 
Exame de Papanicolaou nunca realizado de acordo com algumas variáveis sócio-econômicas, demográficas, ginecológicas e de utilização de serviços de saúde. São Leopoldo, Rio Grande do Sul, Brasil, 2003.

\begin{tabular}{|c|c|c|c|c|c|}
\hline \multirow[t]{2}{*}{ Variável } & \multicolumn{2}{|c|}{ Nunca fez o exame } & \multirow[t]{2}{*}{ Razão de prevalência } & \multirow[t]{2}{*}{ IC95\% } & \multirow[t]{2}{*}{ Valor de $p$} \\
\hline & $\mathrm{n}$ & $\%$ & & & \\
\hline Classe econômica * & & & & & $<0,001$ \\
\hline$A$ e $B$ & 4 & 1,3 & 1,00 & & \\
\hline C & 28 & 8,0 & 5,98 & $2,12-16,85$ & \\
\hline$D$ e $E$ & 34 & 15,6 & 11,62 & $4,18-32,26$ & \\
\hline Escolaridade (anos) & & & & & 0,07 \\
\hline 12 ou mais & 19 & 5,5 & 1,00 & & \\
\hline $8-11$ & 16 & 11,2 & 2,04 & $1,08-3,86$ & \\
\hline $5-7$ & 16 & 7,7 & 1,40 & $0,74-2,67$ & \\
\hline $0-4$ & 15 & 8,9 & 1,62 & $0,84-3,11$ & \\
\hline Renda (salários mínimos) * & & & & & 0,02 \\
\hline$>6,01$ & 4 & 5,4 & 1,00 & & \\
\hline $3,01-6,00$ & 3 & 2,5 & 0,45 & $0,10-1,98$ & \\
\hline $1,01-3,00$ & 22 & 6,6 & 1,21 & $0,43-3,40$ & \\
\hline$<1,01$ & 36 & 11,3 & 2,08 & $0,76-5,67$ & \\
\hline Idade (anos) * & & & & & $<0,001$ \\
\hline $20-29$ & 39 & 15,7 & 1,00 & & \\
\hline $30-39$ & 12 & 5,2 & 0,33 & $0,18-0,62$ & \\
\hline $40-49$ & 12 & 4,8 & 0,30 & $0,16-0,57$ & \\
\hline $50-60$ & 3 & 2,2 & 0,14 & $0,05-0,45$ & \\
\hline Cor da pele & & & & & 0,21 \\
\hline Branca & 52 & 7,1 & 1,00 & & \\
\hline Não branca & 14 & 10,5 & 1,49 & $0,85-2,60$ & \\
\hline Estado civil & & & & & 0,005 \\
\hline Casadas ou em união & 40 & 6,3 & 1,00 & & \\
\hline Viúvas, separadas ou solteiras & 26 & 11,4 & 1,81 & $1,13-2,90$ & \\
\hline Número de filhos & & & & & $<0,001$ \\
\hline Nenhum & 25 & 15,2 & 1,00 & & \\
\hline $1-3$ & 28 & 4,8 & 0,31 & $0,19-0,52$ & \\
\hline 4 ou mais & 13 & 11,5 & 0,76 & $0,41-1,42$ & \\
\hline Idade da menarca (anos) & & & & & 0,20 \\
\hline $8-11$ & 10 & 6,0 & 1,00 & & \\
\hline $12-13$ & 34 & 8,7 & 1,45 & $0,73-2,86$ & \\
\hline 14 ou mais & 22 & 7,1 & 1,19 & $0,58-2,46$ & \\
\hline Uso do preservativo & & & & & 0,17 \\
\hline Sim & 24 & 9,5 & 1,00 & & \\
\hline Não & 42 & 6,8 & 0,72 & $0,44-1,16$ & \\
\hline História familiar de câncer de colo do útero & & & & & 0,16 \\
\hline Não & 62 & 7,9 & 1,00 & & \\
\hline Sim & 4 & 4,6 & 0,58 & $0,22-1,55$ & \\
\hline Consultas médicas no último ano & & & & & $<0,001$ \\
\hline 5 ou mais & 21 & 6,5 & 1,00 & & \\
\hline $3-4$ & 12 & 6,4 & 0,98 & $0,50-1,95$ & \\
\hline $1-2$ & 12 & 4,8 & 0,74 & $0,37-1,47$ & \\
\hline Nenhuma & 21 & 19,3 & 2,95 & $1,68-5,20$ & \\
\hline Tipo de serviço de saúde & & & & & 0,001 \\
\hline Sistema público & 12 & 12,4 & 1,00 & & \\
\hline Seguros e sistema privado & 6 & 2,9 & 0,24 & $0,09-0,61$ & \\
\hline
\end{tabular}

* Teste de tendência linear. 
Análise multivariada para exame de Papanicolaou nunca realizado de acordo com algumas variáveis sócio-econômicas, demográficas, ginecológicas e de utilização de serviços de saúde. São Leopoldo, Rio Grande do Sul, Brasil, 2003.

\begin{tabular}{|c|c|c|c|}
\hline Variável & Razão de prevalência & IC95\% & Valor de $\mathrm{p}$ \\
\hline Classe econômica * & & & $<0,001$ \\
\hline$A$ e $B$ & 1,00 & & \\
\hline C & 5,11 & $1,81-14,39$ & \\
\hline$D$ e $E$ & 9,03 & $3,21-25,38$ & \\
\hline Idade (anos) * & & & $<0,001$ \\
\hline $20-29$ & 1,00 & & \\
\hline $30-39$ & 0,41 & $0,22-0,76$ & \\
\hline $40-49$ & 0,40 & $0,22-0,75$ & \\
\hline $50-60$ & 0,20 & $0,64-0,65$ & \\
\hline Cor da pele * & & & 0,65 \\
\hline Branca & 1,00 & & \\
\hline Não branca & 1,14 & $0,65-2,01$ & \\
\hline Estado civil * & & & 0,16 \\
\hline Casadas ou em união & 1,00 & & \\
\hline Viúvas, separadas ou solteiras & 1,38 & $0,88-2,16$ & \\
\hline Número de filhos ** & & & 0,08 \\
\hline Nenhum & 1,00 & & \\
\hline $1-3$ & 0,55 & $0,19-1,54$ & \\
\hline 4 ou mais & 3,06 & $0,75-12,42$ & \\
\hline Consultas médicas no último ano ** & & & $<0,001$ \\
\hline 5 ou mais & 1,00 & & \\
\hline $3-4$ & 1,05 & $0,53-2,07$ & \\
\hline $1-2$ & 0,73 & $0,38-1,42$ & \\
\hline Nenhuma & 2,57 & $1,52-4,35$ & \\
\hline Tipo de serviço de saúde ** & & & 0,29 \\
\hline Sistema público & 1,00 & & \\
\hline Seguros e sistema privado & 0,60 & $0,23-1,54$ & \\
\hline
\end{tabular}

* Variáveis que ingressaram no primeiro nível e que foram ajustadas entre si;

** Ajustadas para a classe econômica e idade.

As mulheres jovens e as mais velhas, de baixa renda e baixa escolaridade, aquelas classificadas como não brancas, viúvas, solteiras ou separadas realizaram menos o exame preventivo de câncer do colo uterino.

O estudo de Pinho et al. 18 aponta diferentes motivos que levam as mulheres de classes econômicas mais baixas, de menor escolaridade e as mais jovens a buscarem a realização do exame. Diferente das demais que procuram realizar o exame por indicação de profissionais elas geralmente buscam a realização do exame quando apresentam algum sintoma ginecológico, ou necessidade obstétrica 18 .

Portanto, apesar da elevada cobertura de realização do exame citopatológico de colo uterino constatada, no presente estudo ainda observaram-se fatores associados à iniqüidade e falta de integralidade da assistência. 


\section{Resumo}

Este estudo transversal teve por objetivos verificar a cobertura de realização do exame preventivo de câncer do colo do útero e os fatores associados na população de mulheres de 20 a 60 anos residentes na zona urbana de São Leopoldo, Rio Grande do Sul, Brasil, durante o ano de 2003. A amostra foi por conglomerados. Foram analisados dois desfechos, a realização de exame preventivo do câncer do colo do útero nos últimos três anos e exame preventivo de Papanicolaou nunca realizado. Entre as 867 mulheres entrevistadas, 741 (85,5\%; IC95\%: 83,1-87,8) tinham realizado o exame citopatológico do colo uterino nos últimos três anos, 60 (6,9\%; IC95\%: 5,2-8,6) estavam com o procedimento atrasado e 66 (7,6\%; IC95\%: 5,8-9,4) nunca o haviam realizado. Na regressão de Poisson foi observada significância para as variáveis: classe econômica, idade, cor da pele, estado civil. Observou-se que apesar da cobertura elevada, os fatores de risco para o câncer de colo uterino não motivaram a realização do exame.

Esfregaço Vaginal; Neoplasias do Colo do Útero; Cobertura de Serviços de Saúde

\section{Colaboradores}

J. S. Dias-da-Costa e M. T. A. Olinto elaboraram o projeto de pesquisa, participaram da análise e redação do artigo. D. K. Muller e A. M. H. Luz colaboraram na análise e redação do artigo.

\section{Agradecimentos}

Financiado pela Fundação de Amparo à Pesquisa do Estado do Rio Grande do Sul (FAPERGS) via Proad2 (no. 02/06459) e Edital Universal 6 do Conselho Nacional de Desenvolvimento Científico e Tecnológico (CNPq; nº. 473478/200-0), com apoio da Secretaria Municipal de Saúde de São Leopoldo.

\section{Referências}

1. Secretaria de Assistência à Saúde, Ministério da Saúde/Instituto Nacional de Câncer. Atlas de mortalidade por câncer no Brasil 1979-1999. Rio de Janeiro: Instituto Nacional de Câncer; 2002.

2. Kalakun L, Bozzetti MC. Evolution of uterine cervical cancer mortality from 1979 to 1998 in the State of Rio Grande do Sul, Brazil. Cad Saúde Pública 2005; 21:299-309.

3. Coordenação de Prevenção e Vigilância, Instituto Nacional de Câncer. Falando sobre o câncer do colo do útero. Rio de Janeiro: Instituto Nacional de Câncer; 2002.

4. Hatch KD, Fu YS. Câncer cervical e vaginal. In: Berek JS, organizador. Tratado de ginecologia. Rio de Janeiro: Editora Guanabara Koogan; 1998. p. 887-95.

5. Ministério da Saúde/Instituto Nacional de Câncer. Programa Nacional de Controle do Câncer do colo do útero e de mama. Rio de Janeiro: Instituto Nacional de Câncer; 1998.

6. Periodicidade de realização do exame preventivo do câncer do colo do útero. Rev Bras Cancerol 2002; 48:13-5.
7. Leal MC, Gama SGN, Frias P, Szwarcwald CL. Healthy lifestyles and access to periodic health exams among Brazilian women. Cad Saúde Pública 2005; 21 Suppl 1:S78-88.

8. Instituto Brasileiro de Geografia e Estatística. Pesquisa Nacional por Amostra de Domicílios: acesso e utilização de serviços de saúde, 2003. http://www.ibge.gov.br/home/estatistica/ populacao/trabalhoerendimento/pnad2003/ saude/saude2003.pdf (acessado em 13/Nov/2007).

9. Day NE. The epidemiological basis for evaluation of different screening policies. In: Hakama M, Miller AB, Day NE, editors. Screenig for cancer of the uterine cervix. Lyon: International Agency for Research on Cancer; 1986. p. 149-60.

10. Olinto MTA, Dias-da-Costa JS, Mendes KG. A saúde de São Leopoldo: relatório 2000. São Leopoldo: Universidade do Vale do Rio dos Sinos; 2003.

11. Associação Nacional de Empresas de Pesquisa. Critério de classificação econômica Brasil. São Paulo: Associação Nacional de Empresas de Pesquisa; 2003. 
12. Barros AJD, Hirakata UM. Alternatives for logistic regression in cross-sectional studies: an empirical comparision of models that directly estimate the prevalence ratio. BMC Med Res Methodol 2003; $3: 21$.

13. Victora CG, Huttly SR, Fuchs SC, Olinto MTA. The role of conceptual frameworks in epidemiological analysis: a hierarchical approach. Int J Epidemiol 1997; 26:224-47.

14. Dias-da-Costa JS, D’Elia PB, Manzolli P, Moreira MR. Cobertura do exame citopatológico na cidade de Pelotas, RS, Brasil. Rev Panam Salud Pública 1998; 3:308-13.

15. Dias-da-Costa JS, Olinto MTA, Gigante DP, Menezes AMB, Macedo S, Borba AT, et al. Cobertura do exame citopatológico na cidade de Pelotas, Rio Grande do Sul, Brasil. Cad Saúde Pública 2003; 19:191-7.

16. Quadros CAT, Victora CG, Dias-da-Costa JS. Coverage and focus of a cervical cancer prevention program in southern Brazil. Rev Panam Salud Pública 2004; 16:223-32.

17. Hackenhaar AA, Cesar JA, Domingues MR. Exame citopatológico de colo uterino em mulheres com idade entre 20 e 59 anos em Pelotas, RS: prevalência, foco e fatores associados à sua não realização. Rev Bras Epidemiol 2006; 9:103-11.
18. Pinho AA, França Junior I, Schraiber LB, D’Oliveira AFPL. Cobertura e motivos para a realização ou não do teste de Papanicolaou no Município de São Paulo. Cad Saúde Pública 2003; 19 Suppl 2: S303-13.

19. Martins LFL, Thuler LCS, Valente JG. Cobertura do exame de Papanicolaou no Brasil e seus fatores determinantes: uma revisão sistemática da literatura. Rev Bras Ginecol Obstet 2005; 27:485-92.

20. World Health Organization. Cytological screening in the control of cervical cancer: technical guidelines. Geneva: World Health Organization; 1988.

21. Cesar JA, Horta BL, Gomes G, Houlthausen RS, Willrich RM, Kaercher A, et al. Fatores associados à não realização de exame citopatológico de colo uterino no extremo Sul do Brasil. Cad Saúde Pública 2003 ; 19:1365-72.

22. Nascimento CMR, Eluf-Neto J, Rego RA. Pap test coverage in São Paulo municipality and characteristics of the women tested. Bull Pan Am Health Organ 1996; 30:302-12.

Recebido em 21/Ago/2007

Versão final reapresentada em 26/Fev/2008

Aprovado em 06/Mar/2008 